Théologiques

Théologiques

\title{
« Faire communauté ». Comment les Églises fabriquent leurs convertis?
}

\section{Loïc Le Pape}

Volume 21, numéro 2, 2013

La conversion

URI : https://id.erudit.org/iderudit/1028464ar

DOI : https://doi.org/10.7202/1028464ar

Aller au sommaire du numéro

\section{Éditeur(s)}

Faculté de théologie et de sciences des religions, Université de Montréal

ISSN

1188-7109 (imprimé)

1492-1413 (numérique)

Découvrir la revue

Citer cet article

Le Pape, L. (2013). « Faire communauté ». Comment les Églises fabriquent leurs convertis ? Théologiques, 21(2), 101-120. https://doi.org/10.7202/1028464ar
Résumé de l'article

Cet article vise à explorer et à questionner le fonctionnent d'une institution religieuse dans sa capacité à faire communauté, c'est-à-dire à former et à façonner des croyants. Ce travail s’appuie sur des recherches menées lors de mon doctorat, qui présentait une analyse comparative des modalités de la conversion dans les trois religions monothéistes dans la France contemporaine : judaïsme, catholicisme et islam. L'intérêt d'une telle comparaison permet de qualifier le travail des « institutions religieuses " (au sens large) face aux « nouveaux convertis ». En référence aux travaux de J. Lagroye, nous verrons quelle est la réalité de ce travail, en supposant qu'une institution doit « faire communauté ». Pour cela, elle doit imposer une vision générale de l'adhésion et de l'appartenance, en mettant à distance les émotions des convertis et en imposant du banal. Nous pourrons alors conclure sur la tension intrinsèque des institutions religieuses, face à la question de la conversion et l'impératif de "faire communauté » : utiliser les émotions pour mieux les faire oublier. 


\title{
«Faire communauté». Comment les Églises fabriquent leurs convertis?
}

\author{
Loïc Le PAPE* \\ Sciences des religions \\ CNRS, Université d'Aix-Marseille (France)
}

\begin{abstract}
S'engager, ou s'investir, dans une institution, c'est finalement découvrir ce qu'on peut légitimement y faire, ce qu'on peut attendre de ses activités et de son fonctionnement - tel qu'il est et non tel qu'on l'a rêvé avant de "franchir

le pas». Celles et ceux qui font directement cette expérience - les "convertis" dans le cas d'une Église, mais tout autant le jeune officier récemment sorti de l'École d'Application ou le nouvel adhérent d'un parti - rencontrent en quelque sorte, la force de l'objectivé. Ils peuvent certes jouer avec certaines règles, infléchir les

pratiques en fonction de leurs désirs, voire inventer de nouvelles manières de tenir un rôle; il leur faut surtout s'accommoder des exigences du groupe et de ses chefs. (Jacques Lagroye, La vérité dans l'Église catholique, 2006, p. 17)
\end{abstract}

\footnotetext{
Loïc Le Pape est chercheur contractuel à l'IDEMEC (Université d'Aix-Marseille CNRS) où il coordonne, avec K. Boissevain, le projet ANR Circulations religieuses et ancrages méditerranéens (Cirelanmed). Ses recherches portent sur les mobilisations et les controverses politiques à référent religieux, sur les conversions religieuses et plus généralement sur les liens entre politique et religions, dans l'aire méditerranéenne. Il a coordonné (avec Fanny Colonna) la publication de (2010) Traces, désirs de savoir et volonté d'être. L'après-colonie au présent, Actes Sud, et va publier, fin 2014, Une autre foi. Conversions religieuses en France: itinéraires juifs, chrétiens et musulmans.
}

C Revue Théologiques 2013. Tout droit réservé. 


\section{Introduction}

Cet article se propose d'examiner une tension, souvent négligée dans l'analyse sociologique des conversions religieuses contemporaines: le travail de la nouvelle Église (l'institution d'accueil) face à l'effervescence émotionnelle du converti. La religion est aujourd'hui très souvent vécue sur un mode sensible, voire sentimental, et cette dimension s'exacerbe dans le cadre des conversions religieuses. S'il est incontestable qu'il existe de l'émotion en religion (Champion et Hervieu-Léger, 1990), cette émotion doit cependant être "canalisée " par l'institution. Le rôle de celle-ci est de «rendre banal» le changement de religion. À travers l'association de ces mots et verbes contradictoires, nous souhaitons qualifier le travail de l'institution de manière plus générale et aussi plus réflexive. Nous situerons ce travail dans l'espace des tensions que constituent ces contradictions: d'une part entre la suggestion (de l'émotion) et l'imposition (du banal), et d'autre part entre l'exceptionnel (de l'émotion) et le quotidien (le banal). Notre hypothèse est que les Églises doivent "routiniser » les conversions, c'est-àdire les inscrire dans un parcours institutionnel partageable, dans une «institutionnalité». Or, cette « routinisation» passe d'abord par la mise à distance de la ferveur des convertis et par son encadrement dans la quotidienneté de l'Église. En ce sens, nous défendrons l'idée que les Églises accueillent de l'exceptionnel, mais imposent du banal (à l'exception, peutêtre de certaines Églises charismatiques et évangéliques).

Focaliser notre étude sur le travail de façonnage de l'institution d'accueil nous oblige à un réductionnisme théorique revendiqué. Celui de confronter des institutions, les Églises d'accueil (au centre de cet article) à des convertis qui ont témoigné de leurs expériences. Cette façon d'opposer des individus à une institution peut paraître brutale, mais nous revendiquons cet angle d'approche pour deux raisons. Tout d'abord, parce que c'est en ces termes que la question a émergé de nos recherches, à la fois par le discours des convertis et celui des "convertisseurs ", les hommes et les femmes de l'institution. Ensuite, parce qu'un tel angle de vue est le seul à autoriser une focale sur les interactions signifiantes de notre problématique, les façons de faire communauté. Ce travail est le fruit d'une thèse de doctorat, qui opérait une comparaison des processus de conversion, dans les trois monothéismes dans la France contemporaine: le judaïsme, le catholicisme et l'islam (Le Pape 2014, à paraître). Nous avons recueilli, entre 2002 et 2006, une soixantaine de récits de conversion et interrogé 
une trentaine de "convertisseurs ", qu'ils soient laïcs (des accompagnateurs catholiques ou musulmans) ou qu'ils soient clercs (rabbins, pasteurs, curés, imams). Nous aborderons dans cet article les émotions ressenties et partagées par les convertis (des états de grâce, des expériences de communication avec le divin, des bouleversements intimes, des sentiments de plénitude, etc.) et leur façon de les vivre dans leur nouvelle religion. Cette «institution d'accueil» est incarnée par des individus (clercs ou séculiers), inscrits dans des configurations sociales particulières (le manque de "professionnels", les rapports contradictoires au sein des religions sur le sens de la conversion) et dans des processus historiques généraux (un contexte de sécularisation, la perte d'influence des Églises et un héritage historique spécifique à chaque culte). L'institution doit donc être définie par avance comme plurielle, potentiellement contradictoire et bien plus labile qu'il n'y paraît.

La définition de travail retenue de l'institution est proche de celle que proposait Durkheim: "On peut appeler institution toutes les croyances et tous les modes de conduite institués par la collectivité; la sociologie peut alors être définie: la science des institutions, leur genèse et leur fonctionnement » (Durkheim 1986, XXII). Pour être un peu plus précis, je m'inscris dans la lignée de Jacques Lagroye qui applique la notion d'institution à l'Église catholique:

Lorsqu'ils étudient un grand rassemblement d'acteurs sociaux que des activités communes mettent en relation réglée et plus ou moins continue - une administration, un corps d'armée, un parti politique - , les chercheurs sont incités à parler d'institution s'ils veulent rendre compte de tout ce qui pèse, en un sens, sur ces acteurs. L'usage du terme "institution" signifie alors qu'ils accordent la plus grande attention à ce qui est institué ou "objectivé » de diverses manières, à tout ce qui est par conséquent susceptible d'assurer un minimum de cohérence dans les conduites des membres, d'unifier partiellement leurs façons de se comporter, voire de modeler leurs conceptions du juste et de l'inique, du licite et de l'inacceptable, du bien et du mal, du «vrai» et du faux. (Lagroye 2006, 12-13)

C'est dans cette perspective socio-anthropologique que nous appelons "clercs» toute personne ayant une forme d'autorité (savante ou administrative), au sein de l'institution. De la même façon, le terme Église (et son pluriel) sera pris dans son sens anthropologique, tel que Durkheim le définissait: l'Église est une communauté morale qui unit. Durkheim définit en effet la religion et, dans la seconde partie de la définition, précise la notion d'Église: "Une religion est un système solidaire de croyances et de pratiques relatives à des choses sacrées, c'est-à-dire séparées, interdites, 
croyances et pratiques qui unissent en une même communauté morale, appelée Église, tous ceux qui y adhèrent» (Durkheim 1985, 65). Notre recherche s'inscrit donc dans une sociologie comparative des institutions (Lagroye et Offerlé 2011), ici des institutions religieuses comparables, malgré les avertissements de Levent Tezcan (2008): "In social sciences, when speaking about religious group/community (congregation) one actually has the Church as model in mind [...]». Nous comparons trois cultes en France, car ils sont ancrés dans une tradition (un rapport historique et politique avec la République qui a toujours «organisé» ses cultes sur le même schème) et qu'ils obéissent, pour la conversion religieuse, à un fonctionnement quasi identique relevant de la sociologie des institutions: la gestion d'un changement d'état, au sein et sous le contrôle de l'institution elle-même.

Nous avons vu en guise d'introduction ce que j'entends par institution religieuse et les termes de la comparaison. Nous verrons dans une première partie l'importance de l'émotion, dans les processus de conversion contemporains, et nous discuterons des principales approches de la sociologie des religions. Dans un deuxième temps, nous qualifierons le travail de l'institution qui doit assurer une gestion des émotions et, dans le même temps, institutionnaliser la diversité des parcours. Puis, dans une troisième partie, nous verrons que c'est la «routinisation » de la conversion qui est recherchée, malgré des exceptions. Nous pourrons alors conclure sur la tension intrinsèque des institutions religieuses, face à la question de la conversion et l'impératif de «faire communauté »: utiliser les émotions pour mieux les faire oublier.

\section{De l'émotion en conversion}

Un même constat semble partagé par les chercheurs en sociologie des religions: l'émotion est très valorisée, dans les formes modernes du croire et dans les façons de les analyser. En effet, ce thème a connu un engouement lorsque les spécialistes des religions ont voulu caractériser, dans les années 1960 et 1970, l'apparition des nouvelles formes religieuses qui faisaient davantage appel à l'expérience personnelle. Ainsi, pour qualifier les renouveaux charismatiques, les pentecôtismes, etc., les sociologues se sont massivement tournés vers des explications de type émotionnel.

These types of popular movements, for example, early Methodism or Pentecostalism, mobilizes religious responses; but it also disciplines those responses, eradicates random superstition, rationalizes understanding and 
commitment, and works for the steady reduction of immanentism. These movements [...] provide a new education of emotions. (Wilson 1989, 152)

On retrouve ce constat sous différentes formes et différentes plumes: Livet propose une analyse érudite des émotions (2002), tandis qu'elles sont discutées par Paperman et Ogien (2002) et mobilisées de manière un peu excessive par Kirkpatrick et Shaver (1990) pour montrer que la vie religieuse de l'adulte est en partie dépendante des conditions émotionnelles du lien à ses parents. Dans le même temps, dans d'autres disciplines des travaux ont également mobilisé une sociologie des émotions, notamment en science politique (Braud 1996 ou Traïni 2009) ou en anthropologie (Terrain 1994).

\subsection{Que faire des renouveaux émotionnels?}

En France, un ouvrage a été consacré à la question, suscitant quelques vives réactions (Champion et Hervieu-Léger 1990; Favret-Saada 1994). La contribution de D. Hervieu-Léger, minutieuse analyse de la place des émotions dans la sociologie de Durkheim et de Weber, nous servira de guide de lecture. L'hypothèse principale de Hervieu-Léger est double: la réactivation émotionnelle contemporaine peut traduire, soit la remise en cause de l'encadrement bureaucratique des Églises (l'émotion comme protestation socioreligieuse), soit la fin de l'interprétation ecclésiale d'un discours religieux qui serait devenu inaudible, l'émotion étant alors un «langage au-delà du langage» (1990, 245). Détaillons progressivement cette double hypothèse.

La première hypothèse est issue de l'examen des textes de Durkheim, qui considère la religion comme une effervescence collective. En parlant de "conversion protestataire ", Danièle Hervieu-Léger reprend à son compte la traditionnelle distinction de deux expériences religieuses: le mythe de l'expérience fondatrice (le religieux plein) et l'institutionnalisation qui en résulte (les Églises) à travers la domestication de l'émotion par un langage et «l'intégration de l'expérience dans un dispositif symbolique spécifique». Elle explique sa démonstration en quatre points:

Une première proposition pose que «l'émotion des profondeurs", qui se trouve à la source de toutes les expressions religieuses de l'humanité [...] constitue, par nature, la forme originaire, la plus pleine, la plus dense, de l'expérience religieuse en général; une seconde proposition est que l'institutionnalisation implique nécessairement l'euphémisation, l'affadissement, la routinisation et même la dénaturation de cette expérience; une troisième proposition tient au fait que cette institutionnalisation ne 
constitue pas seulement un résultat, mais aussi un facteur actif de la perte sociale de la religion $[. .$.$] ; une quatrième et dernière proposition$ liant structurellement le processus d'institutionnalisation et le mouvement par lequel une religion s'installe dans une fonction d'attestation de l'ordre social, donne à toutes les ré-invocations de l'expérience émotionnelle des commencements la signification d'une protestation socioreligieuse. (Hervieu-Léger 1990, 228-229)

Cette dimension protestataire est contenue dans le fait que la réactivation émotionnelle s'apparenterait à une critique de la «froideur des institutions religieuses» (ibidem), voire à une critique sociopolitique vis-à-vis d'un monde sécularisé.

La seconde hypothèse découle de l'interprétation wébérienne, pour qui, toujours selon Danièle Hervieu-Léger, " "la rationalisation progressive des conceptions éthico-religieuses et l'élimination des représentations magiques primitives" implique logiquement la "désémotionnalisation" de l'univers religieux, l'euphémisation progressive et l'élimination tendancielle des états altérés de conscience qui sont associés à l'expérience primitive du divin» $(1990,237)$. En ce sens, la rationalisation, «inséparable de l'institutionnalisation de la sphère religieuse », n'annule pas l'expérience émotionnelle qui se trouve "remaniée, transformée, résumée sous d'autres formes» (241).

Danièle Hervieu-Léger estime donc que la réactivation de l'émotion, dans des formes religieuses contemporaines (charismatiques, évangéliques), peut être considérée comme une protestation face à la domination rituelle, mais aussi une critique des discours exégétiques des Églises institutionnalisées. Elle peut également être le symptôme de recompositions plus larges de la sécularisation occidentale: les formes de religions "émotionnelles» viendraient concurrencer une histoire (donc des symboles et références religieuses) qui ne se transmet plus.

Dans un ouvrage postérieur, Danièle Hervieu-Léger (1999) complète ses propositions, en faisant de la "dimension émotionnelle» l'un des éléments de l'identification religieuse. Elle identifie quatre «dimensions typiques de l'identification»: la dimension communautaire («les marques sociales et symboliques qui définissent les frontières du groupe religieux»), la dimension éthique (l'" acceptation par l'individu des valeurs attachées au message religieux porté par une tradition particulière»), la dimension culturelle ( «l'ensemble des éléments cognitifs, symboliques et pratiques qui constituent le patrimoine d'une tradition particulière») et enfin, la dimension émotionnelle («le sentiment de "fusion des consciences" ou "l'émotion des 
profondeurs" [...] ressort premier et fondateur de l'expérience religieuse ») (Hervieu-Léger 1994, 72-74).

La combinaison de ces quatre dimensions en pôles et l'identification de deux tensions (la tension entre dimensions éthique et communautaire et la tension entre dimensions culturelle et émotionnelle) constituent l'identification religieuse. La focalisation ou l'autonomisation vers l'un de ces pôles entraîne la sortie de la religion.

Une chose essentielle, pour l'examen que nous menons du travail de l'institution, consiste à poser comme son travail propre la nécessité de régler les tensions entre ces quatre dimensions: «celles-ci se construisent comme identité confessionnelle lorsque l'identification à une tradition religieuse particulière implique l'acceptation des conditions d'identité (communautaires, éthiques, culturelles et émotionnelles) fixées - ou au moins délimitées - par l'institution qui s'en présente comme le garant. Dans ce cas - longtemps le plus courant - l'articulation de ces quatre dimensions est réglée par l'institution elle-même. C'est elle en particulier qui assure, au moins idéalement, le maintien d'un équilibre entre les logiques contradictoires qui placent ces dimensions en tension entre elles » (Hervieu-Léger 1994, 77). C'est en ce sens que ces dimensions contradictoires peuvent nous intéresser, car nous analysons ici le travail de régulation que mène l'institution entre l'émotion individuelle et le sentiment collectif.

\subsection{Remettre l'émotion à sa place?}

Que nous apporte alors de tirer aujourd'hui la ficelle théorique de l'émotion en religion? On l'a vu, Danièle Hervieu-Léger l'utilise pour expliquer des changements dans la modernité religieuse. Bien que ses hypothèses soient pertinentes, j'aimerais en proposer un usage plus restreint. Je me place pour cela dans la lignée d'Albert Piette et des critiques qu'il adresse aux travaux qui mobilisent les émotions dans des contextes historiques postulés comme différents. Il pense que cette façon de concevoir l'émotion s'apparente à une surinterprétation: "Quand il s'agit du discours catastrophiste des sociologies de la modernité, assimilé à une mythologie de la décadence ou de la mutation, la surinterprétation, qui devient alors mépris de son temps par rapport à un autre temps plus plein, est le fait d'une ignorance de l'histoire et de la méconnaissance de la quotidienneté " (Piette 2003, 8). Ce constat relève d'un autre sens sociologique: porter attention aux formes de jugement dites «altérées» (par l'émotion, par les sentiments), 
c'est refuser de voir ce qui est sous les yeux, ce qui se donne à voir comme des faits et gestes parmi les plus banals. Ce que Piette appelle le quotidien, et que nous pourrions subsumer sous la formule de Fanny Colonna: « une ethnographie au ras du sol» (Colonna 2004). Pour autant, je ne m'engage pas à "mettre à distance " cette émotion, contrairement à ce que suggère Piette, car elle est centrale dans l'expérience de la conversion religieuse. Tout comme sont centraux les efforts de distanciation qu'opère l'institution, face à ces émotions.

Alors que retenir de l'émotion des convertis? J'ai délibérément opté pour un pragmatisme méthodologique, me bornant à entendre les émotions que les convertis me soumettaient. Je n'y attachais pas de jugement ni ne sollicitais les convertis à leur propos, mais ne fermais pas la discussion non plus. On m'a confié, lors de plusieurs entretiens, des histoires d'émotions, de rencontres, de moments de félicité, de signes divins sur lesquels je ne peux pas faire l'impasse. Ces récits d'émotions me projetaient en plein dans le double dilemme du sociologue (et de l'anthropologue): que faire de ces expériences? Quel statut méthodologique leur accorder ? Pour répondre à la première question, j'ai pris de la distance avec les théories de l'émotion, sans chercher à donner une interprétation à ces expériences. Ce que les convertis ont bien voulu me faire partager n'est donc pour moi, ni la résurgence d'une quelconque sacralité des profondeurs ni la manifestation personnalisée de l'émotion du collectif. Ce sont simplement des expériences personnelles, des événements intimes livrés lors d'une interaction. Ce partage était souvent signe de confiance ou, tout du moins, de succès de l'échange. L'important est de concevoir l'émotion comme quelque chose d'éminemment personnel et subjectif et, en même temps, d'essayer, autant que possible, de voir quelles sont ses inscriptions dans le social (où et quand apparaît l'émotion ?). Le travail des sociologues serait alors de replacer l'émotion du côté du social, sans chercher à la minimiser dans les retranscriptions du vécu individuel. Ainsi, je n'ai pas cherché à compléter telle ou telle théorie à partir de ces sentiments, pas plus qu'à valider ou infirmer les propos de Danièle Hervieu-Léger. Devant le statut «incertain » de ces moments, j'ai décidé de privilégier la description plutôt que la théorisation. Me semble plus importante la façon dont les convertis me racontaient ce moment et ce qu'ils liaient à ça. Privilégier une sorte de faisceau de références, d'actes d'élocution, plutôt que prendre l'émotion comme base théorique. S'attacher à ce qu'ils en disent, à voir comment ces moments sont réactivés, racontés, est à mon sens plus pertinent que de décortiquer cette émotion. 
Ce choix implique donc une posture méthodologique particulière. Puisqu'il ne peut y avoir ni observation ni participation, et que mon travail ne se contente pas d'énumérer ces émotions, j'ai pris le parti de retranscrire ces moments, à travers les façons dont les convertis en parlaient: en faisant le lien avec le travail de l'institution. Nous allons donc voir comment l'Église se saisit de ces émotions et comment son travail est de la mettre à distance, tout en développant un discours valorisant sur ces «sentiments».

\section{Les Églises entre émotion et institutionnalisation: canaliser l'émotion}

Le rôle des Églises est de mettre à distance l'émotion des convertis, qui s'avère peu compatible avec l'inscription dans une institution. Ce n'est pas la transformation d'une quelconque "émotion des profondeurs» qui est en jeu, mais davantage le passage de sentiments individuels et subjectifs à une foi raisonnée et collective. Là est le point central de l'activité de l'institution: favoriser le passage de l'émotion individuelle au sentiment collectif. Sans cette émotion (appel, évidence, certitude, etc.), il serait difficile d'adhérer au collectif. C'est par un travail sur l'émotion individuelle que peut se construire le sentiment collectif. Soutenir l'hypothèse de la canalisation de l'émotion n'est pas, pour autant, accréditer l'idée que les Églises proposent un religieux «froid». Bien au contraire, nous essaierons de montrer, d'un point de vue néo-institutionnaliste, que les clercs valorisent cette émotion, cet apprentissage personnel des sentiments religieux. L'émotion est donc «l'accroche" par laquelle l'institution peut façonner ou «fabriquer» le nouveau converti. C'est par la domestication des émotions qu'on accède à la généralisation et au passage au collectif. Néanmoins, au niveau individuel, elles restent valorisées, les «joies délirantes de l'expérience» (William James) étant le terreau de cette transformation.

\subsection{Ancrer la démarche dans une communauté}

L'étape la plus délicate dans le dialogue avec les futurs convertis est, pour les clercs, de généraliser des trajectoires, autrement dit, de prendre l'ensemble des postulants à la conversion et d'en faire un bloc. C'est le moyen le plus simple de passer du cheminement personnel à l'expérience collective. J'ai assisté à des réunions où, lorsque des clercs parlaient aux candidats, ils s'attachaient à reconnaître les histoires personnelles pour les relier ensuite à un collectif, à une communauté: 
Vous tous qui allez vous convertir, inch'allah, vous avez des histoires différentes, des passés différents, des motivations différentes. Finalement, vous êtes tous différents. Mais vous vous rejoignez sur votre volonté d'embrasser une nouvelle croyance. Devenir musulman, cela peut se faire très rapidement, mais vous avez choisi d'approfondir votre foi, vos croyances. Ce qui est bien, c'est que vous êtes tous ensemble ici comme une petite partie de la communauté. Vous comprenez? Ce qui est important, ce n'est pas que vous êtes tous différents, c'est de vous rendre compte que nous formons là une petite partie de la communauté. C'est la communauté qui est importante. D'accord? (Réunion de candidats et convertis à l'islam, Marseille)

Ce qui est à noter dans ce type de démarche, c'est une montée en généralité du discours des clercs, dans l'adresse aux convertis: ils doivent faire le lien entre les histoires personnelles, qu'ils connaissent et entretiennent (par des contacts personnalisés), et l'histoire de la nouvelle religion. Ce «jeu d'échelles» (Revel 1996) est systématiquement entrepris pour embô̂ter une trajectoire dans une autre, plus large et profondément historique. Le résultat escompté est une transformation de l'idéal de la conversion, de la valorisation d'un parcours personnel vers l'adhésion à une communauté, plus large.

Pour mener à bien ce travail de mise en équivalence, il y a tout d'abord le discours des clercs-formateurs. Ils associent aux trajectoires des convertis des caractéristiques générales qui permettent déjà de penser le processus de conversion comme quelque chose de collectif. Cette façon de dire se retrouve dans des entretiens: les discussions portaient sur la conversion en général et sur les actions que les clercs mènent auprès des convertis. Dans le judaïsme, la conversion est fondamentalement liée à une communauté, à un peuple. Tout converti est alors questionné, puis formé, en référence à ce peuple, à son histoire et aussi à ses persécutions :

Mon enseignement, c'est un universalisme à travers une spécificité bien définie. Comme ma femme dit toujours, le judaïsme c'est la religion de l'effort, et c'est évident qu'on ne peut pas demander à l'humanité de faire la même chose. Simplement, on demande à des gens de faire un effort particulier pour s'élever. Attention, ça ne veut pas dire que les autres ne le font pas, il n'y a pas d'exclusive! Je ne considère pas qu'il y ait une supériorité à être juif. Le Talmud dit d'ailleurs: "ton sang est plus rouge que le sien». Le problème, c'est que cet universalisme, à travers cette spécificité bien définie, qui a quand même 3500 ans, possède des contraintes énormes. Alors, quand quelqu'un veut se convertir, le Talmud dit qu'il faut lui demander: qu'est-ce 
que tu as besoin d'aller te fourrer avec des gens qui sont persécutés constamment? (M. A., rabbin et enseignant à des convertis, en Alsace)

Il y a ensuite le discours des personnes chargées d'accompagner le parcours des candidats à la conversion. Que ce soient des enseignants dans le judaïsme, des accompagnateurs dans le catholicisme ou des imams dans l'islam, tous mobilisent une pratique qui privilégie le collectif au détriment (ou à la place) de l'individuel. Nous prendrons en exemple un extrait d'entretien avec un accompagnateur catholique de Strasbourg:

[Le processus de conversion], c'est un itinéraire baptismal dans l'Église. Cet itinéraire comprend des évangiles qui expliquent bien la relation qu'on devrait avoir, en théorie, avec Lui. Personnellement, je suis très sensible à ça... Le religieux, ça peut être tout et n'importe quoi... Je ne voudrais pas être un gourou qui impose tout, je préfère garder une façon de se poser des questions et accepter d'être parfois un peu perdu. Il faut [que le catéchumène] se fabrique son Dieu, qu'il trouve ses marques, qu'il crée sa croyance. Et aussi, comme je suis d'un certain âge, les relations avec un homme jeune, on a quand même un certain vécu, une expérience, une façon de concevoir la vie, et c'est souvent bénéfique pour eux, comme de les structurer un peu, humainement. Parce que la religion n'est pas seulement de l'ordre de l'affectif ou je ne sais pas quoi, un genre de distribution de grigris pour éviter la scoumoune, c'est pas ça, Christ n'a jamais dit "pas de scoumoune ». Alors, ça aussi, vous voyez, comme on doit tout de suite au départ voir que chaque chose est possible ou pas. Mais on essaye aussi de faire parler ces catéchumènes, et c'est pas une mince affaire [...] Oui c'est très important, et aussi de voir le niveau, parce qu'une tentation majeure de l'accompagnateur serait de lui en mettre plein la vue, de lui asséner des vérités, et c'est vrai que j'étais technicien, vous aussi à l'université vous avez votre jargon, l'Église a aussi un jargon, et qui est parfois assez hermétique. Et il y a des mots clefs qu'il faut prendre la peine d'expliquer: la crainte de Dieu ne veut pas dire tremblement, respecter Dieu aussi, mais ça ne veut pas dire que nous sommes rien ou que Dieu est tout, mais ça veut dire qu'il y a là une relation dans l'altérité, et c'est cette altérité qu'on doit expérimenter. (Henri, accompagnateur laïc dans l'Église, Strasbourg)

À travers ces extraits d'entretiens, nous allons nous attacher à comprendre les logiques permettant à l'accompagnateur de proposer (et faire accepter ?) l'idée du collectif. Nous avons identifié deux façons de le faire, valables dans les trois cultes étudiés.

Il faut, tout d'abord, expliquer le collectif par la «technique ecclésiale». J'entends par là les références aux textes (ici l'évangile), à la façon 
d'en parler, aux mots (le jargon), etc. Le parcours et les expériences de chacun sont relus et retraduits, en fonction d'un vocabulaire spécifique, des mots (apostat, giyyur, kafir), des expressions. Il y a généralisation puis rationalisation par le vocabulaire. Mais au-delà des mots, la technique ecclésiale, c'est aussi des situations et des objets: apprendre, sous la direction d'un clerc à faire la prière collectivement, manipuler des objets religieux, considérer le statut du livre saint, enlever ses chaussures, se prosterner. Dans tous ces exemples, il y a des séquences d'action qui mettent en rapport un corps et un environnement. Apprises à plusieurs, ces séquences d'actions permettent aux clercs d'accélérer l'apprentissage du collectif.

Il faut ensuite convaincre de l'importance de l'expérience personnelle. Ce qui paraît a priori comme contradictoire s'inscrit en fait dans une technique de l'exemple: en prenant son propre parcours, l'accompagnateur montre les erreurs à ne pas commettre, les fausses pistes et les interprétations erronées. On constate ci-dessus que l'accompagnateur se sert de son expérience personnelle pour accentuer le travail du candidat à la conversion dans sa construction à la communauté. D'autres discussions nous ont conforté dans cette idée, comme l'emploi des erreurs, de la narration des égarements de celui qui transmet: "Ne faites pas la même erreur que celle que j'ai faite. » C'est d'ailleurs une forme historique de récit que de raconter des anecdotes qui parlent d'échecs pour prévenir les erreurs futures.

L'utilisation combinée de ces deux techniques est le moyen qu'emploient les clercs pour faire oublier aux convertis leurs émotions et ancrer leur corps dans une pratique, des techniques, dans un environnement d'humain et d'objets. Il est une autre façon de s'éloigner de ces sentiments, c'est tout simplement de les relativiser, en regard d'un argument indiscutable, d'une irréfutabilité: la parole de Dieu.

\subsection{Mettre à distance les émotions trop fortes}

Le travail des clercs est de convaincre de l'importance primordiale du collectif, face à un converti et à son parcours individuel. Les choses peuvent devenir délicates lorsque les candidats arrivent «pleins d'émotions» difficilement contrôlables ou lorsqu'ils ont une attitude de méfiance envers l'institution, ce qui n'est pas rare. Ces bouffées spirituelles comme ces réticences, il appartient de ne pas les nier, tout en les intégrant au cheminement institutionnel de l'individu. C'est ici qu'apparaît alors la troisième technique, celle de l'argument irréfutable. Ce moyen d'imposer au converti 
une rupture, en douceur, d'avec ses émotions, suppose de partir de cellesci. Souvent, les individus arrivent avec des sentiments difficilement maîtrisables. C'est ici que l'opération prend tout son sens: comment objectiver puis domestiquer un sentiment irrationnel, sans le nier?

Nous avons donc observé que les clercs, dans ces cas-là, passent par «la volonté de Dieu ». Certes, l'expression paraît galvaudée. Et pourtant, elle leur est utile, car elle permet d'imposer un type de lecture qui ne peut que susciter l'adhésion, y compris chez les plus émus des convertis. Cette étape est primordiale, car les clercs disposent, dans la volonté de Dieu, d'un argument de poids, irréfutable. En imposant des jugements, ils peuvent faire varier les opinions et les idées (donc les sentiments et les émotions) des convertis. Dans l'exemple précédent, l'accompagnateur donne une interprétation de ce que n'aurait jamais dit Jésus Christ ( "pas de scoumoune») ou de la bonne façon de croire en Dieu («la crainte de Dieu ne veut pas dire tremblement $»$ ).

Est-ce à dire, en allant plus loin, que nous revenons aux arguments de Danièle Hervieu-Léger, en les faisant nôtres? Y aurait-il un sentiment religieux, une émotion des profondeurs, d'un côté, et, de l'autre, une institutionnalisation impliquant une perte?

Cette vision d'un religieux à deux vitesses, où à deux "paliers " a connu, dans le champ de l'anthropologie et de la sociologie des religions, une fortune qui déborde très nettement les usages qui en ont été faits dans le courant durkheimien. Dans une version souvent simplifiée, elle commande une approche, largement répandue, de l'institutionnalisation comme «domestication » d'une expérience religieuse émotionnelle, envisagée elle-même à la fois comme expérience "primitive " (c'est-à-dire comme "forme élémentaire» de la religion, d'un point de vue chronologique), et comme une expérience "originaire" (c'est-à-dire comme forme fondatrice du sentiment religieux, d'un point de vue génétique). (Hervieu-Léger 1990, 221-222)

Je reste pourtant réticent à toute analyse portant sur les différences de nature entre l'émotion et l'institutionnalisation. J'ai simplement constaté que parfois, des convertis arrivent avec des émotions personnelles (dont ils ne savent d'ailleurs que faire) et que l'institution s'arrange pour ne pas les voir, les susciter, et parfois, en leur appliquant la technique de «la volonté de Dieu ». Cela n'est pas une émotion différente. Elle n'est simplement pas totalement calibrée pour être dite dans l'institution et c'est en ce sens que les techniques évoquées ci-dessus sont efficaces: il ne faut pas nier l'émotion du converti, sans pour autant l'entretenir. C'est donc à un travail 
subtil de polissage ou de calibrage que se livrent les clercs, sans aucunement atténuer les émotions, leurs forces et leur variété. En ce sens, l'institution impose du banal, tout en gardant la reconnaissance de l'exceptionnel.

\section{Ce que l'Église attend du converti : conversion et routinisation}

Une fois la célébration de la conversion effectuée - qu'elle ait été shahada, bain rituel dans le mikvé et circoncision ou baptême - comment se passent les premiers pas de l'individu désormais converti dans sa nouvelle Église? En nous appuyant sur les témoignages des convertis et parfois des clercs, j'aimerais proposer une idée qui est relativement ancrée dans les pratiques et fait l'objet d'un consensus tacite: la tentation de l'oubli de la conversion. Autrement dit: une fois converti, il faut oublier que l'on a été converti. En effet, le statut des convertis étant ce qu'il est — trahison puis adhésion une logique de l'oubli s'impose qui préside à la conversion. Une exception existe, me semble-t-il, dans le protestantisme évangélique, où la réactivation et le rappel du «sauvetage» ou de la «rencontre», puis du baptême, est très importante. C'est un mode de fonctionnement institutionnel que Sébastien Fath appelle «religion de conversion» (Fath 2004). Pour autant, dans les trois cultes qui nous intéressent ici, cela n'entraîne pas que chaque conversion doive nécessairement être masquée, cachée et qu'une fois effectuée elle soit tue. Nous nous situons là dans un temps plus long que celui de l'élaboration du récit et de sa publicisation.

\subsection{La conversion et son oubli: la conversion est son oubli}

Il peut apparaittre paradoxal de soutenir que la réussite de la conversion est son oubli, alors que cet article suppose l'accueil des émotions, leur façonnage puis leur revendication dans l'institution. Cependant, la dimension temporelle est importante. L'élaboration du récit effectuée, la conversion narrée et expliquée aux proches, une fois le converti reconnu en tant que tel, il entre progressivement dans une phase de routinisation et, partant, de l'oubli graduel de la conversion (pour une analyse relationnelle de la conversion, voir Le Pape 2013 et 2014). Réussir sa conversion, c'est réussir à la faire oublier: idéalement, le converti doit alors se fondre dans la communauté et passer comme un croyant parmi d'autres.

Ça, c'est quelque chose de très important dans le judaïsme, parce que moi on m'a dit qu'après ma conversion, je devrais me faire oublier. Mais tel quel 
on me l'a dit. J'ai très bien compris et c'est ce que je fais, mais ils ont vraiment la trouille! Enfin bon, pardon, je ne devrais pas dire ça! (Maëva, convertie au judaïsme)

C’est dans le judaïsme que la logique est poussée au maximum puisque, nous venons de le voir, il est parfois demandé aux convertis de se faire discrets. Cette prescription est à mettre en lien avec l'autodéfinition du groupe et la façon dont il conçoit la possibilité de les intégrer. Plus il est délicat d'entrer dans un groupe, plus les impétrants doivent se faire discrets. Pourtant cette discrétion est aussi de mise dans d'autres cultes, mais cette fois, de l'initiative des convertis:

Non, ça se passe très bien à la mosquée, ça y est. Maintenant, je tâche de me faire discret, d'arriver à l'heure ou un tout petit peu en avance, et faire comme tout le monde, au moment où tout le monde le fait. C'est ma nouvelle façon de faire! Ça fait un peu mouton, mais tu sais, au départ, c'est pas évident d'aller à la mosquée. (Jamal, converti à l'islam)

Cette discrétion me semble dictée par la mauvaise image que peuvent avoir les convertis, dans leur nouvelle communauté. Les accueils difficiles sont assez fréquents, car le converti véhicule l'image sulfureuse de celui qui a trahi ses croyances précédentes et qui arrive empli d'émotions et de bonne volonté, sans aucune idée précise de sa future religion. À la suite de la publicisation de la conversion, la tendance est donc à une routinisation qui s'opère, entre autres, par une attitude plutôt discrète. L'émotion devient subjective, elle est cachée ou dissimulée. Mais nous allons voir qu'il y a des exceptions et que parfois, l'exposition des émotions est tolérée.

\subsection{L’exception: être célèbre (ou célébré)}

Dans quelle(s) situation(s) une Église peut-elle contredire l'idée selon laquelle la conversion est son oubli ? Cette question est en somme l'hypothèse de Frédéric Gugelot, à propos des récits de conversion au catholicisme des intellectuels du début du $\mathrm{Xx}^{\mathrm{e}}$ siècle. Il montre que les témoignages d'écrivains et d'intellectuels célèbres sont utilisés par l'Église catholique (avec l'assentiment des convertis) comme publicité et, dans un contexte de laïcisation, comme littérature «de combat». Du côté de l'Église catholique, ces récits vont être utilisés pour recruter de nouveaux fidèles dans un contexte de laïcisation:

La sécularisation de la société, la privatisation de la foi, le sentiment de persécution qui habite alors le catholicisme conduisent à favoriser la mise 
en exergue de la figure du converti. La postérité de Péguy dans les années 1930, alors qu'il n'a pas achevé son parcours vers le catholicisme, prouve son actualité. Leur volonté de se rattacher au passé pour mieux justifier leur propre parcours masque la modernité de leur cheminement, et son signe, «l'autonomie du sujet-croyant », et permet leur promotion. (Gugelot 2002, 62)

Malgré le risque anachronique, un point commun demeure: aujourd'hui encore, dans les trois cultes, subsistent des figures, des personnalités qui, converties récemment, sont «utilisées » comme références. Régulièrement exposées publiquement, ces personnalités font état de leur conversion, ont parfois publié des récits de leur cheminement et parlent de leurs émotions de croyants, tout comme les intellectuels catholiques des années 1920. Prenons un exemple dans chaque culte, tout en notant qu'à la différence des années 1920, ce ne sont pas forcément les Églises qui mettent en avant les témoignages des nouveaux convertis. Point de vertus apologétiques désormais, mais des témoignages dans la presse et à la télévision qui donnent à voir des retours à la religion, parfois spectaculaires.

Dans le judaïsme, ces personnalités existent, mais elles restent cependant assez discrètes. La cantatrice Nathalie Dessay s'est convertie au judaïsme, pour des raisons «matrimoniales ", même si ses réponses sont souvent plus compliquées. Elle parle ainsi de sa conversion:

Après avoir épousé Laurent Naouri, chanteur lui aussi, vous vous êtes convertie au judaïsme? - Ce n'était pas une démarche spirituelle, car je suis agnostique. Mais une façon de le reconnaître pleinement comme mon mari. Moi qui viens d'un catholicisme plein de certitudes, j'aime chez les juifs cette culture de la question, ce travail à l'infini sur les textes, ces exégèses perpétuelles sur trois petites lignes. Génial. Comme en musique, on ne vient jamais à bout du détail. (Interview accordée au journal Télérama, n² 2968, 2 décembre 2006)

Du côté du catholicisme, ce sont essentiellement des écrivains, des penseurs, qui font état de leur récente conversion et dont les parcours deviennent publics. La particularité du catholicisme est que ces intellectuels possèdent le plus souvent une origine catholique, ils partagent «ce vieux fond de cuve » qu'est une culture chrétienne, selon l'expression de HervieuLéger. Pourtant, régulièrement, un intellectuel bascule en religion ou revient à la foi de ses ancêtres. L'un des convertis célèbres récents est l'écrivain Maurice Dantec, dont la personnalité, les options politiques et les écrits controversés rajoutent un "piquant médiatique» à ses interviews. 
Mais également Jean-Claude Guillebaud (auteur de Comment je suis redevenu chrétien), René Girard, Michel Serres, Jacques Ellul ou Max Gallo.

Dans l'islam également, de nombreuses conversions sont publicisées. Citons pour mémoire des sportifs célèbres, basketteurs, comme Olivier Saint-Jean (Tariq Abdul Wahad); footballeurs, comme Nicolas Anelka ou Franck Ribéry; chanteurs comme Abd al Malik (2004), Akhenaton ou Cat Stevens. Dans certains de ces exemples, les convertis rechignent à parler de leur nouvelle vie. C'est le cas des footballeurs qui préfèrent rester discrets. D'autres, au contraire, n'ont pas de problèmes à raconter et à témoigner de leur conversion, comme Cat Stevens ou Tariq Abdul-Wahad: "Je te le dis, et c'est pour moi comme une ultime conviction, comme une certitude: mon destin, c'était l'islam» (Islam de France, 6, p. 25).

Ces expositions ne sont pas utilisées par les Églises comme une littérature "de combat». Les récits de conversion sont présentés comme des témoignages personnels, d'un choix individuel et d'un accomplissement de soi. C'est là la grande différence avec les récits de Frédéric Gugelot. Pour aller plus loin, nous pourrions supposer que ces histoires sont la partie immergée de l'iceberg religieux de nos sociétés contemporaines: si des personnes célèbres reviennent à la religion, nombre d'inconnus, a fortiori, peuvent faire les mêmes choix. En ce sens, les gens célèbres (ou célébrés) font eux aussi communauté.

\section{Conclusion}

Nous avons voulu dans cet article explorer une tension qui apparaît comme constitutive de toute conversion dans une religion instituée: l'émotion individuelle du converti et le «faire communauté» de l'institution. En comparaison, le protestantisme évangélique (et peut-être les «nouveaux mouvements religieux ») sont fondés sur la valorisation de l'émotion et de l'extraordinaire. Au sein de ces groupes, c'est l'émotion individuellement ressentie et collectivement partagée qui fait et fonde la communauté. Les sentiments autour de la perception du destin personnel, de la grâce, de l'effervescence sont mis en valeur. C'est peut-être l'une des particularités des religions instituées que de banaliser et de routiniser ce processus, en fonction des étapes que nous avons vues dans cet article. Il n'en reste pas moins que toute conversion, qu'elle soit vers des Églises instituées ou de nouveaux mouvements religieux, naît dans une émotion forte. Une conversion qui ne prendrait pas sa source dans cette émotion ne pourrait pas aboutir à un sentiment d'appartenance collective. 


\section{Références}

Aвd Al Malik (2004), Qu'Allah bénisse la France, Paris, Albin-Michel.

Braud, P. (1996), L'émotion en politique, Paris, Presses de Sciences-Po.

Champion, F. et Hervieu-Léger, D. (1990), De l'émotion en religion. Renouveaux et traditions, Paris, Centurion.

Colonna, F. (2004), Récits de la province égyptienne. Une ethnographie sud-sud, Paris, Actes Sud.

Durkheim, E. (1985) [1912], Les formes élémentaires de la vie religieuse, Paris, PUF.

1986 [1895], Les règles de la méthode sociologique, Paris, PUF.

Fabre, P.-A. (1999), «Présentation. Conversions religieuses: histoires et récits ", Annales HSS, 54/ 4, p. 805-812.

Fath, S. (2004), dir., Le protestantisme évangélique, un christianisme de conversion? Entre ruptures et filiations, Turnhout, Brépols.

Favret-SaAda, J. (1994), «Weber, les émotions et la religion », Terrain, 22, disponible sur <http://terrain.revues.org/3088>.

Gugelot, F. (1998), La conversion des intellectuels au catholicisme en France 1885-1935, Paris, C.N.R.S. Éditions.

(2002), "Le temps des convertis, signe et trace de la modernité religieuse au début du $\mathrm{xx}^{\mathrm{e}}$ siècle", Archives de sciences sociales des religions, 119, p. 45-64, disponible sur: <http://assr.revues.org/document1724.html>.

Hervieu-Léger, D. (1990), «Renouveaux émotionnels contemporains. Fin de la sécularisation ou fin de la religion?", dans F. Champion et D. Hervieu-Leger, De l'émotion en religion. Renouveaux et traditions, Paris, Centurion, p. 217-248.

(1999), Le pèlerin et le converti. La religion en mouvement, Paris, Flammarion.

James, W. (2001) [1902], Les formes multiples de l'expérience religieuse, Chambéry, Exergue; titre original: (1960) The varieties of Religious Experience. A Study in Human Nature, Londres, Collins.

Kirkpatrik, L.-A. et Shaver, P.- R. (1990), "Attachment Theory and Religion: Childhood Attachments, Religious Beliefs, and Conversion ", Journal for the Scientific Study of Religion, 29/3, p. 314-334. 
Lagroye, J. (2006), La vérité dans l'Église catholique. Contestations et restaurations d'un régime d'autorité, Paris, Belin.

(2009), Appartenir à une institution. Catholiques en France aujourd'bui, Paris, Economica.

Lagroye, J. et Offerle, M. (2011), dir., Sociologie de l'institution, Paris, Belin.

Le Pape, L. (2005), "Les récits de conversion: d'une histoire personnelle romancée à l'analyse sociologique d'un engagement religieux ", dans Alfa 2005, Maghreb et sciences sociales, 1, p. 77-87.

(2013), "Converts at Work. Confessing a Conversion", dans N. Marzouki, et O. Roy, dir., Religious conversions in the Mediterranean world, Londres, Palgrave/Macmillan, p. 98-114.

(2014, à paraître) Une autre foi. Conversions religieuses en France: itinéraires juifs, chrétiens et musulmans, Aix-en-Provence, Presses Universitaires de Provence.

Livet, P. (2002), Émotions et rationalité morale, Paris, PUF.

Paperman, P. et Ogien, R. (1995), dir., La couleur des pensées. Sentiments émotions, intentions, Paris, Éditions de l'EHESS (Raisons Pratiques 6).

Piette, A. (2003), Le fait religieux. Une théorie de la religion ordinaire, Paris, Economica.

Revel, J. (1996), dir., Jeux d'échelles: la micro-analyse à l'expérience, Paris, Gallimard.

Les émotions (1994), Terrain 22, disponible sur <http://terrain.revues. org/652>.

Tezcan, L. (2008), "Governmentality, Pastoral Care and Integration ", dans A. Al-Hamarneh, et J. Thielmann, dir., Islam and Muslims in Germany, Leiden, Brill, p. 117-132.

Traini, C. (2009), Émotions..., mobilisations!, Paris, Presses de sciences-po.

Weber, M. (1995) [1971], Économie et société, 2 t., Paris, Pocket.

Wilson, B. (1989) [1982], Religion in sociological perspective, Oxford, Oxford University Press. 


\section{Résumé}

Cet article vise à explorer et à questionner le fonctionnent d'une institution religieuse dans sa capacité à faire communauté, c'est-à-dire à former et à façonner des croyants. Ce travail s'appuie sur des recherches menées lors de mon doctorat, qui présentait une analyse comparative des modalités de la conversion dans les trois religions monothéistes dans la France contemporaine: judaïsme, catholicisme et islam. L'intérêt d'une telle comparaison permet de qualifier le travail des "institutions religieuses» (au sens large) face aux "nouveaux convertis». En référence aux travaux de J. Lagroye, nous verrons quelle est la réalité de ce travail, en supposant qu'une institution doit «faire communauté ». Pour cela, elle doit imposer une vision générale de l'adhésion et de l'appartenance, en mettant à distance les émotions des convertis et en imposant du banal. Nous pourrons alors conclure sur la tension intrinsèque des institutions religieuses, face à la question de la conversion et l'impératif de "faire communauté »: utiliser les émotions pour mieux les faire oublier.

\section{Abstract}

This paper studies how a religious institution is able to create a feeling of community, that is to say making and shaping believers. This study is based on my doctoral research, namely a comparative analysis of conversion mechanisms in the three monotheistic religions in contemporary France: Judaism, Catholicism, and Islam. Such a comparison has the advantage to show how "religious institutions" (largely speaking) deal with their new converts. In reference to J. Lagroye's research, this paper shows what is really at work - taking into account that an institution must create a feeling of community. As a consequence, it must enforce a common vision of membership and belonging by pushing away the converts' emotions and focusing on triviality. Some conclusions are drawn concerning the paradox that characterizes religious institutions when it comes to conversion and belonging: using emotions so they can be forgotten more easily. 